J. Sustain. Wireless Syst.

Vol.02/ No. 1

Pages: $42-49$

http://irojournals.com/irosws/

DOI: https://doi.org/10.36548/jsws.2020.1.005

\title{
5G Systems with Low Density Parity Check based Chanel Coding for Enhanced Mobile Broadband Scheme
}

\author{
Dr. Joy Iong Zong Chen, \\ Professor, Department of Electrical Engineering, \\ Da-Yeh University, \\ Taiwan. \\ Email id: jchen@mail.dyu.edu.tw
}

\begin{abstract}
The 5G mobile communication standard based radio access technology (RAT) is analysed for implementation of several candidate coding schemes in this paper. The third generation partnership project (3GPP) in the 5G scenario based on the Enhanced mobile broadband (eMBB) scheme is considered. Factors like flexibility, complexity of computation, bit error rate (BER), and block error rate (BLER) are considered for the purpose of evaluation of the coding schemes. In order to evaluate the performance various applications and services, a suitable set is of parameters are provided. The candidate schemes considered for this purpose are polar codes, low density parity check (LDPC) and turbo codes. Fair comparison is performed by investigation of block lengths and obtaining suitable rates by proper design. In an additive white Gaussian noise (AWGN) channel, the performance of BLER / BER is obtained for diverse block lengths and code rates based on simulation. The simulation results show that the performance of LDPC is relatively efficient for various code rates and block lengths despite the better performance of polar codes at short block lengths. As an added advantage, LDPC codes also offer relatively low complexity.
\end{abstract}

Keywords: 3GPP; polar codes; LDPC; 5G; eMBB;

\section{Introduction}

Cellular communication systems and the services offered by them has been enhanced and evolved from generation to generation since the first to fourth generation (1G-4G). The voice call services of $1 \mathrm{G}$ was enhanced to provide text messaging services in the second generation $(2 \mathrm{G})$. Further, an addition of mobile internet services has lead to the evolution of third generation (3G) technology. The current $4 \mathrm{G}$ technology offers better services when compared to $3 \mathrm{G}$ technologies up-to 250 times in terms of mobile data rate of $100 \mathrm{Mbps}$ and stationary data rate of $1 \mathrm{Gbps}$. The upcoming evolution in the mobile technology is the 5G New Radio (NR) [1] that has promising features and wider usability in comparison to $4 \mathrm{G}$. All aspects of 5G technology is proposed to be several times more efficient and improved than the existing $4 \mathrm{G}$ technology in terms of the performance parameters. Despite the lack of standardization of $5 \mathrm{G}$ performance parameters, the network latency of the typical parameters ranges around 10 Gigabits per millisecond and the capacity of the network is multiplied by tens and thousands times the current network. Low cost and power consumption are also desired. Along with these features, machine to 
J. Sustain. Wireless Syst.

Vol.02/ No. 1

Pages: $42-49$

http://irojournals.com/irosws/

DOI: https://doi.org/10.36548/jsws.2020.1.005

machine communication (M2M) interface with ultra high reliability and low cost with a battery life of up to ten years should be supported by the $5 \mathrm{G}$ technology [2]. Coverage, bandwidth, signalling efficiency and spectral efficiency are to be enhanced significantly when compared to $4 \mathrm{G}$ technology in order to facilitate these requirements.

The 5G network technology and its development is mainly driven by two significant trends. Delivery of content-rich services like videos and so on demand higher-capacity and faster wireless broadband services. The need for ultra broadband services is driven by the upsurge of video traffic and applications like virtual reality, live video broadcast, and full high definition (FHD) videos. The evolution of $4 \mathrm{G}$ technology was also based on similar drives whereas, the current $5 \mathrm{G}$ technology demands data rates that offers thousand times greater capacity. The rapid evolution of Internet of things (IoT) also demands the need for 5G technology with internet based massive ultra-low-latency and ultra-reliable connectivity. Applications such as smart homes, smart city, augmented and virtual reality services, wireless health services, utility applications, industrial automation, driver assistance, autonomous driving, transportation systems, vehicle-to infrastructure and vehicle-to vehicle communication requires $5 \mathrm{G}$ technology. The requirements of low data rate applications with high reliability and low latency makes this technology different from the mobile broadband services. These applications also require communication between machines rather than humans. IoT applications also demand lower cost and power consumption.

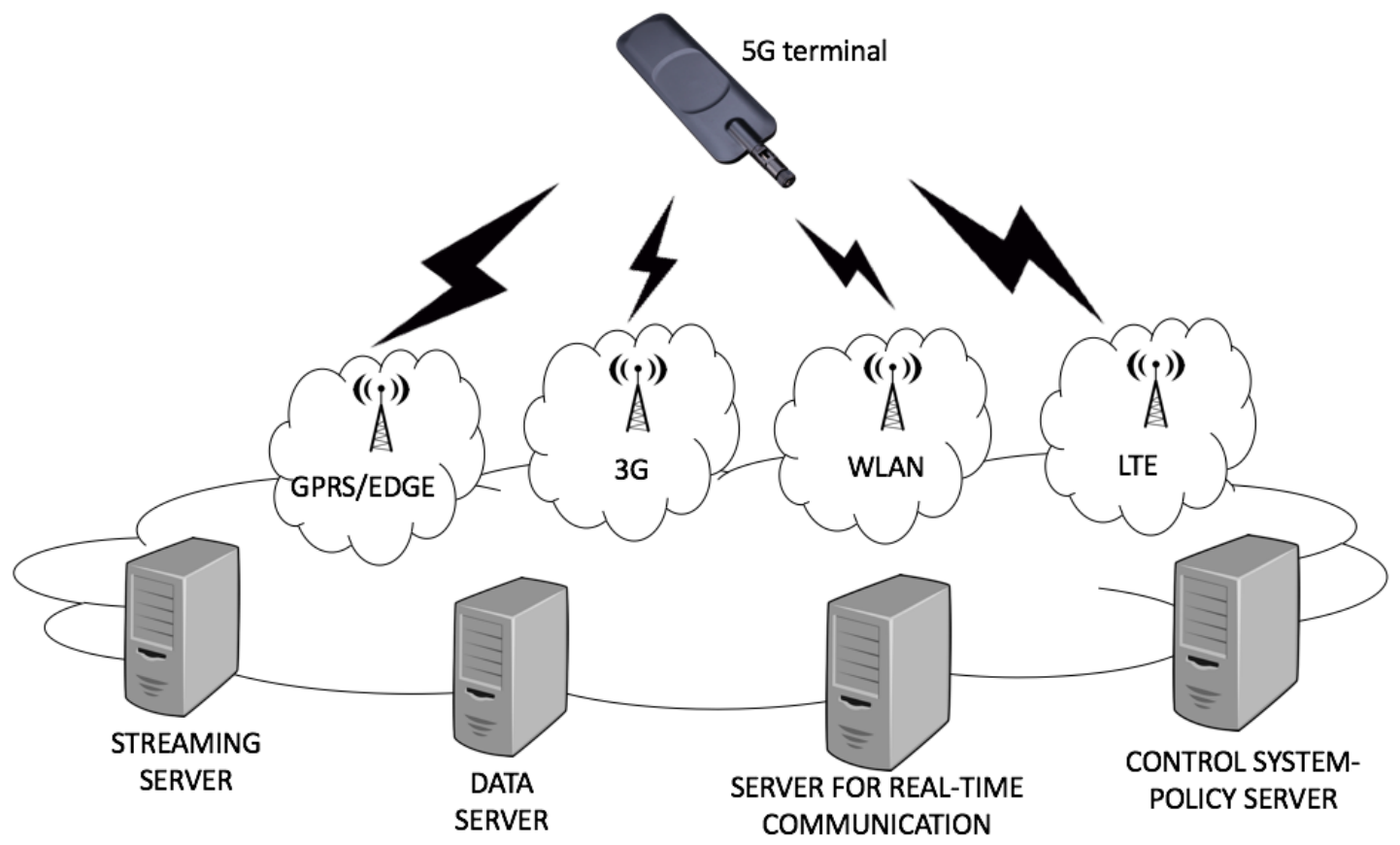

Figure 1: 5G architecture 
J. Sustain. Wireless Syst.

Vol.02/ No. 1

Pages: $42-49$

http://irojournals.com/irosws/

DOI: https://doi.org/10.36548/jsws.2020.1.005

Figure 1 represents the basic architecture of 5G technology in which there are various servers for streaming, storage of data, real-time communication and control system policies and data transmission protocols like 3G, LTE, WLAN and GPRS/EDGE which connects to the 5G terminal. Massive machine type communications (MMTC), Ultra reliable low latency communications (URLLC) and eMBB are the scenarios of the 5G NR access technology. These scenarios can be suited to the requirements of the user. MMTC and URLLC require highly reliable communication based on the usage and are sensitive to latency. Higher capacity and data rates are the requirement of eMBB. eMBB also offers high profitability and strong interface as it is critical and can support the growing demands of the users. In comparison to 4G Long Term Evolution (LTE), the eMBB supports a broad range of modulation orders, code lengths and code rates. In this paper, $1 / 5$ to $8 / 9$ is the code rate range chosen for experimentation and 100 to 8000 bits range of code length are considered.

\section{Literature Review}

In applications as the eMBB that demand wide range of code rates and block lengths, several coding schemes fail to provide good and consistent performance, however, there are several coding schemes that has the capability of attaining improved performance at larger block lengths. For a broad range of code lengths and coding rates, polar [3], LDPC [4] and turbo codes [5] offer promising performance in BLER and is the preferred choice as a physical layer for 5G technology. The feedback decoding rule is used for building a turbo code encoder with the help of the associated decoder and two recursive systematic convolutional codes that are concatenated in parallel. Digital Video Broadcasting (DVB), LTE standards, Universal Mobile Telecommunications System (UMTS), 3G/4G mobile communication and deep space communications make use of turbo codes as it offers a low probability of error along with performance within a $1 \mathrm{~dB}$ fraction from the the Shannon limit. In case of higher data rates, the complexity of implementation is high for the block lengths and code rates of eMBB and the performance requirements are not satisfied despite being used in $3 \mathrm{G}$ and $4 \mathrm{G}$ communication.

Turbo code BER consists of an error floor. Despite being invented in 1962, LDPC codes were not used until Mackay rediscovered it in 1997 [6]. The Shannon limit can be reached by the LDPC codes due to their empirical performance similar to or better than the turbo codes. Low density 010s make the sparse parity check matrix in the linear LDPC codes. The practical and simple decoding algorithms of LDPC codes are the result of the sparse parity check matrix. Iterative belief propagation algorithm is used for decoding operation. The parity checks and bit values are estimated in the check nodes and bits respectively. The values are passed between each iteration. The requirements of the application decide the iteration count and the accuracy of estimates are improved during every iteration. Complexity, latency and bit error performance are the possible trade-off factors. The gain of the decoder can be further enhanced by using soft decision algorithms along with the modern LDPC decoders [7]. 802.16e (Mobile WiMAX), 802.11n (Wi-Fi allowing MIMO) and such communication systems deploy LDPC codes due to their optimized channel capacity at theoretical level. 
J. Sustain. Wireless Syst.

Vol.02/ No. 1

Pages: $42-49$

http://irojournals.com/irosws/

DOI: https://doi.org/10.36548/jsws.2020.1.005

\section{Candidate Coding Schemes}

\subsection{Turbo codes}

This paper performs simulation and discussion of turbo codes using 3GPP LTE standard specification parameters. Two streams of outputs namely the parity stream and systematic stream are generated by each individual convolutional turbo code encoder. The second encoder is fed with the data as an input bit stream that is interleaved. The fundamental decoder component used for parallel concatenated encoding scheme is a MAX-Log-MAP algorithm based turbo decoder that performs iterative decoding. The encoder as well as decoder makes use of identical internal interleavers.

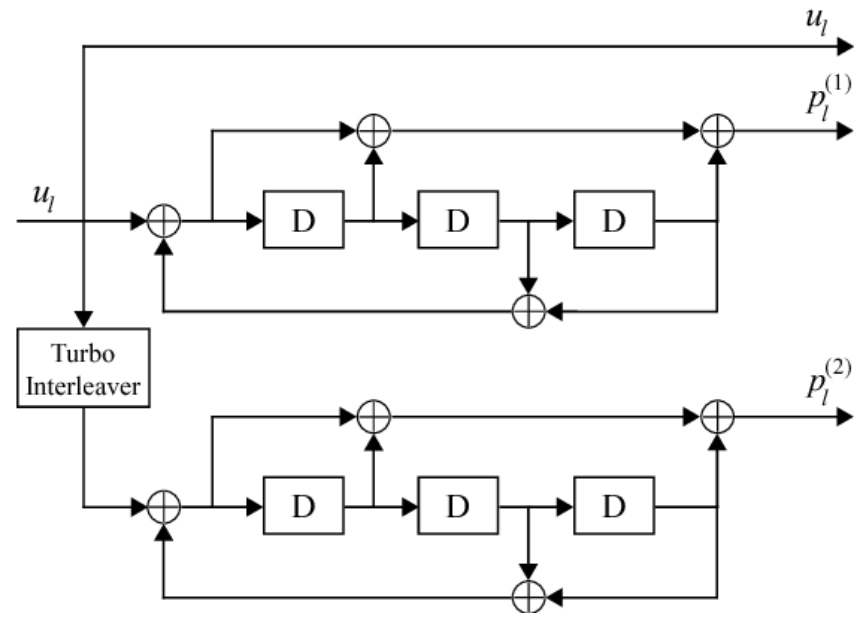

Figure 2: Turbo Encoder using LTE rate 1/3 [8]

\subsection{LDPC codes}

Simulation is performed by means of Quasi-Cyclic LDPC (QCLDPC) codes. A parity check matrix $\mathrm{H}$ is used for characterization of QCLDPC matrix. Circulant permutation matrices or zero matrix is made up of small square blocks. Adjustments in the circulant permutation matrices in $\mathrm{H}$ leads to easy variations in the code length which is a major advantage of QCLDPC code. The computational complexity is reduced with the use of a sub optimal algorithm called min-sum algorithm and the optimal decoder used for LDPC is the sum product algorithm (SPA) 
J. Sustain. Wireless Syst.

Vol.02/ No. 1

Pages: $42-49$

http://irojournals.com/irosws/

DOI: https://doi.org/10.36548/jsws.2020.1.005

\subsection{Polar codes}

$\mathrm{O}(\mathrm{n} \log \mathrm{n})$ is the encoding complexity of Kronecker products on which recursive polar code construction can be performed. Successive cancellation (SC) decoding also offers similar decoding complexity. In order to attain competitive performance in comparison with the available coding schemes, an SC decoder is insufficient. Incorporation of list decoding technique to the traditional LC decoder leads to the creation of SC list (SCL) decoder. With the risk of increased complexity, the performance of the code can be further enhanced using cyclic redundancy check (CRC) bits.

Table 1: Various Coding Schemes and the Percentage of Decoding Complexity

\begin{tabular}{|c|c|c|c|c|c|c|c|c|}
\hline $\begin{array}{c}\text { Block } \\
\text { Length }\end{array}$ & \multicolumn{4}{|c|}{128} & \multicolumn{4}{c|}{2048} \\
\hline $\begin{array}{c}\text { Coding } \\
\text { Scheme }\end{array}$ & Turbo & LDPC & $\begin{array}{c}\text { Polar } \\
\text { SC }\end{array}$ & $\begin{array}{c}\text { Polar } \\
\text { SCL }\end{array}$ & Turbo & LDPC & $\begin{array}{c}\text { Polar } \\
\text { SC }\end{array}$ & $\begin{array}{c}\text { Polar } \\
\text { SCL }\end{array}$ \\
\hline $\mathbf{1 / 3}$ & $100 \%$ & $100.6 \%$ & $1.6 \%$ & $16.7 \%$ & $100 \%$ & $100.6 \%$ & $2.4 \%$ & $23.5 \%$ \\
\hline $\mathbf{1 / 2}$ & $100 \%$ & $59 \%$ & $1.0 \%$ & $11.3 \%$ & $100 \%$ & $59.2 \%$ & $1.7 \%$ & $15.7 \%$ \\
\hline $\mathbf{2 / 3}$ & $100 \%$ & $38 \%$ & $0.9 \%$ & $8.5 \%$ & $100 \%$ & $38 \%$ & $1.3 \%$ & $11.8 \%$ \\
\hline
\end{tabular}

\section{Simulation}

The Quadrature amplitude modulation (QAM) is used for simulation of the candidate coding schemes. Channel state information (CSI) along with AWGN is considered for simulation [9]. Table 1 provides the coding schemes and the evaluation of decoding complexity for the various schemes and ranges of block length. Block lengths of 128 and 2048 are used for analysing the decoders and their algorithmic complexity for various coding schemes. An LTE advanced encoder consisting of a block interleaver and two eight-state constituent encoders is used for turbo codes. Eight iterations with a scaling factor of seven is implemented on a scaled MAX-Log-MAP decoder at the receiver end. Puncturing technique is used for obtaining higher rate codes while the original turbo code has the code rate of $1 / 3$. 
J. Sustain. Wireless Syst.

Vol.02/ No. 1

Pages: $42-49$

http://irojournals.com/irosws/

DOI: https://doi.org/10.36548/jsws.2020.1.005

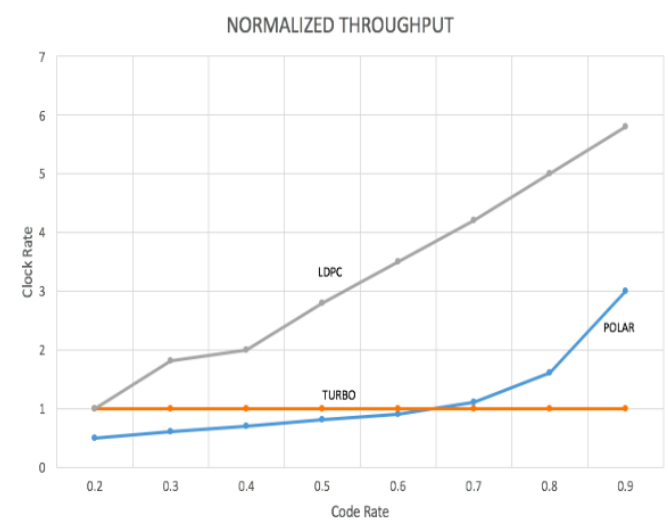

Figure 3: Normalised throughput for various code rates and coding schemes

The simulation of LDPC performs encoding using IEEE 802.1 based parity check matrices. The receiver makes use of OMS decoder with 50 iterations and the offset value of 0.3 . The size of the parity check matrix's base matrix is altered for obtaining varied code lengths. Figure 3 represents the normalised throughput of various code rates and clock rates for the discussed coding schemes. The AWGN channel construction is the base for construction of polar codes [10]. For all lengths and code rates the eight CRC bits and a list size of eight is simulated for the CRC aided-SCL decoder (CRCASCL) to analyse the performance of coding schemes [11].

\section{Results of Simulation}

The coding schemes for various block lengths are analysed and plotted in Figure 4 (a) to provide insights of the polar, LDPC and turbo codes. The ratio of power spectral density $\left(\mathrm{E}_{b}=\mathrm{N}_{0}\right)$ and energy to noise is plotted for coding schemes like BER and BLER. The rates $1 / 2,1 / 2$ and $2 / 3$ are used for analysis of which the polar SCL outperforms the LDPC and turbo codes in case of 128 bits block length. Further, analysis is done by gradually increasing the block length to 512 bits in which it was found that the performance of the polar CRCA-SCL decoder was similar to that of the turbo and LDPC coding schemes. At this rate and block length, in BER, turbo codes perform better than the polar codes despite offering similar performance in BLER. 
J. Sustain. Wireless Syst.

Vol.02/ No. 1

Pages: $42-49$

http://irojournals.com/irosws/

DOI: https://doi.org/10.36548/jsws.2020.1.005

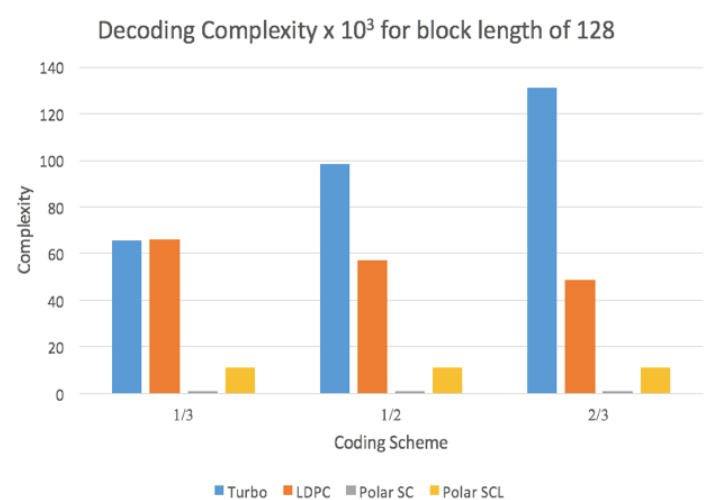

(a)

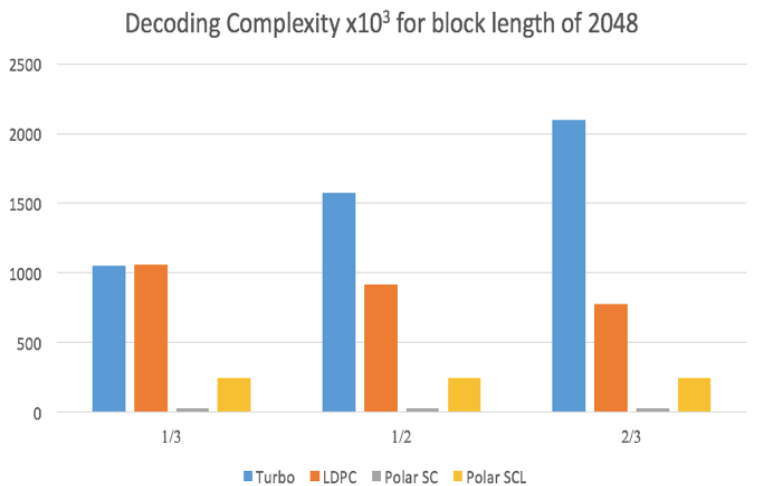

(b)

Figure 4: Analysis of Coding Schemes for the block length of (a) 128 and (b) 2048

At the coding rate of $1 / 3$, the polar codes with LDPC and CRCA-SCL decoders are outperformed by the turbo codes with the increase in block length as represented in Figure 4 (b). In comparison with turbo and polar codes, LDPC offers improved performance for the coding rates $2 / 3$ and $1 / 2$. Despite the unavailability of CRC bits, the LDPC codes offer improved performance. The implementation complexity varies based on the practical factors of the implementation environment and cannot be completely depended on the theoretical assumptions made.

\section{Conclusion}

The performance parameters of 5G New Radio technology is analysed with various candidate coding schemes in this paper. The block lengths are varied and the tests are carried out at block lengths of 124, 512, 1024 and 2048 bits. CRCA-SCL decoder in combination with polar codes offer improved performance for shorter block lengths when compared to LDPC and Turbo codes. Whereas, consistently good performance is provided by LDPC codes for all block lengths and coding rates. Also, CRC is not required for analysing the performance of LDPC codes, but it can be used for enhancing its performance. Turbo and LDPC codes have error floors, however, polar codes have the advantage of the unavailability of error floors. Despite this feature, polar codes are not versatile as it is essential to perform channel based code construction to optimize the performance of polar codes. In order to attain channel independent code design, further research is to be done. 
J. Sustain. Wireless Syst.

Vol.02/ No. 1

Pages: $42-49$

http://irojournals.com/irosws/

DOI: https://doi.org/10.36548/jsws.2020.1.005

\section{References}

[1] Bioglio, V., Condo, C., \& Land, I. (2020). Design of polar codes in 5G new radio. IEEE Communications Surveys \& Tutorials.

[2] Gohil, A., Modi, H., \& Patel, S. K. (2013, March). 5G technology of mobile communication: A survey. In 2013 international conference on intelligent systems and signal processing (ISSP) (pp. 288-292). IEEE.

[3] Schürch, C. (2016, July). A partial order for the synthesized channels of a polar code. In 2016 IEEE International Symposium on Information Theory (ISIT) (pp. 220-224). IEEE.

[4] Brack, T., Alles, M., Lehnigk-Emden, T., Kienle, F., Wehn, N., L'Insalata, N. E., ... \& Fanucci, L. (2007, April). Low complexity LDPC code decoders for next generation standards. In 2007 Design, Automation \& Test in Europe Conference \& Exhibition (pp. 1-6). IEEE.

[5] Sripimanwat, K. (2005). Turbo code applications. Dordrecht: Springer.

[6] Briffa, J. A., \& Schaathun, H. G. (2008, December). Improvement of the Davey-MacKay construction. In 2008 International Symposium on Information Theory and Its Applications (pp. 1-4). IEEE.

[7] Brack, T., Alles, M., Lehnigk-Emden, T., Kienle, F., Wehn, N., L'Insalata, N. E., ... \& Fanucci, L. (2007, April). Low complexity LDPC code decoders for next generation standards. In 2007 Design, Automation \& Test in Europe Conference \& Exhibition (pp. 1-6). IEEE.

[8] 3rd Generation Partnership Project; Technical Specification Group Radio Access Network; Evolved Universal Terrestrial Radio Access (E-UTRA); Physical Channels and Modulation, Release 9, 3GPP TS 36.211 V9.0.0 (2009- 12), Technical Specification.

[9] Romero, M. A. S., \& Soria, F. R. C. (2019). A Comparative Performance Analysis for Spatial Modulation (SM) and Quadrature-Amplitude Modulation (QAM) Techniques. Difu100ci@ Revista en Ingeniería y Tecnología, UAZ, 13(1).

[10] Yan, S., He, B., Cong, Y., \& Zhou, X. (2017, May). Covert communication with finite blocklength in AWGN channels. In 2017 IEEE International Conference on Communications (ICC) (pp. 1-6). IEEE.

[11] Hamad, A. A., Ibrahim, M. K., Al-hayder, A. A., \& Tamkeen, S. A. (2019). Fifth Generation (5G) New Radio (NR) Channel Codes Contenders Based on Field-Programmable Gate Arrays (FPGA): A Review Paper. Journal of University of Babylon for Engineering Sciences, 27(3), 76-83. 